\title{
Campus Intelligence Mental Health Searching System Based on Face Recognition Technology
}

\author{
Zixiang Wang \\ Dujiangyan Bayi Juyuan senior high school, China
}

\begin{abstract}
The phenomenon of teenage campus suicide has become the focus of attention of parents, schools and the society. The causes behind it are extremely complicated, and the root cause is psychological and spiritual problems. However, one's negative psychology is often hidden, and it is difficult to detect and effectively intervene before the tragedy. How to effectively identify students with suicidal tendencies in order to prevent tragedies has aroused extensive research and discussion among the government, academia and the public. Through investigation and research, it is found that the current popular computer cutting-edge technologies such as artificial intelligence and computer vision can be well used for human emotion recognition and behavior prediction, and put into use in schools as a mental health auxiliary diagnosis and treatment system, thus effectively reducing the suicide rate on campus. The scenario assumes that machine learning can be used to deduce the risk of psychological problems in human samples by analyzing the frequency of negative emotions in facial expressions. Based on this, this paper proposes an effective solution for campus suicide prediction, and designs a set of auxiliary diagnosis and treatment system based on campus monitoring network system for suicide behavior prediction and student mental health analysis. Through preliminary experimental analysis and verification, the suicide psychological auxiliary diagnosis and treatment system has achieved good results in face recognition success rate, emotion recognition success rate and behavior prediction success rate. With the input of more experimental data and the increase of self-training time, the prediction system will perform better.
\end{abstract}

Key words: Suicide prevention; Machine learning;
Face recognition; Human emotion recognition

Publication date: July, 2020

Publication online: 31 July, 2020

*Corresponding author: Zixiang Wang, wangyifei@ xyzrgroup.com

\section{Introduction}

\subsection{Social background}

Campus, which should have been filled with happiness has become many students' nightmares. Under heavy pressure from study, family, or being bullied, they go on a road to deliverance for death---suicide!

In May, 2017, a senior high school student in a Shanghai school committed suicide by cutting his wrist after leaving a suicide note of ten thousand. In September, 2017, a student in Junior Three in Jilin committed suicide due to a conflict. In October, 2017, a student in Junior Two in Kailuan committed suicide by jumping from a building due to pressure; In a period of a year, many suicide cases on campus happened one after another nearby. According to the survey on incomplete suicide and having suicide ideas in Anhui, Guangdong, Tianjin and Beijing, the probability of having suicide ideas in middle schools is $13.2 \%$ to $28.0 \%{ }^{[1-5]}$. Behind the data, in addition to the related family's sorrows one after another, people need a reflection. If they can get enough attention from the school and receive professional psychological counseling and intervention, perhaps they can also enjoy the happiness of the world as normal children. However, the teachers are busy, and they are difficult to find out the certain students with suicide ideas among thousands of students. A system for 
mental health diagnosis and treatment was used widely, thereby effectively reducing the suicide rate on campus.

\subsection{Suicide intention among adolescents at home and abroad}

There is a single technique at home and abroad for predicting juvenile suicide, generally based on selfrating scales and questionnaires ${ }^{[6-7]}$. However, due to the time-consuming investigation and randomness, the prediction cannot be performed efficiently, and the maximum accuracy cannot be achieved. There are very few intelligent predicting models for machine learning, but most of them are monitoring public opinions on social platform. But the relevant data obtained based on these methods cannot help effectively and accurately predict suicide ideas. Compared with the above sampling methods and predicting models, the model for predicting suicide ideas by facial expression recognition and emotional analysis in surveillance videos is more accurate and effective, but this technology has not been practically used at home and abroad.

Face recognition technology, such as face comparison technology and the emerging human facial expression recognition technology are quite mature internationally with relatively mature technical detection methods ${ }^{[8]}$.

\subsection{The content and contributions of this paper}

The psychological problem mentioned above is mainly aimed at the prediction of suicide ideas, which refers to the collection and analysis of facial expressions to tell their psychological emotions and other specific behaviors such as tracks of behaviour, etc. Personal data is derived comprehensively. The campus intelligent mental health questioning and screening system based on face recognition technology contains a large amount of information processed by the computer, fast processing, and uninterrupted work to assist the psychologist to discover students of potential suicide on campus. The core innovation of this paper is the structural design of the system for behaviour screening and the working principle of some functions. Facial recognition technology is now relatively mature.

\subsection{Hypothesis of the study}

Based on the existing psychological research results, this paper assumes that the risk of psychological problems such as depression and anxiety in the experimental samples can be derived through machine learning and by analyzing the number and frequency of negative emotions in the facial expressions. Psychological literature shows that depression, anxiety and sudden out-of-group activities are the main factors for high-risk suicide students, so this paper defines the students with depression and anxiety as those with potential suicide ideas ${ }^{[9]}$.

The hypothesis is assumed within the laboratory that if multiple images with emotional characteristics in multiple experimental samples through facial recognition and integral model can successfully distinguishes normal and depressed samples into the constructed system.

\subsection{Introduction of the system}

The pictures taken by all the cameras distributed on campus are transmitted to the monitoring center through the LAN system. While recording the images, the center for data analysis selects the pictures taken by each camera for facial comparison and facial emotional recognition. The data of facial emotions, the place where the picture was taken, and the shooting time are stored in the corresponding personnel file according to the face comparison results.

Samples are marked the recorded places stored on the interval map in the file according to the shooting time from early to late, and then use the behaviour restoration algorithm of low frequency to obtain the users' behaviour tracks. Samples who are out of group activities are judged based on machine learning through the dissociation degree and historical records. At the same time, the emotional data and behaviour tracks stored in the computer are processed by the artificial intelligence system and the dimension chart of mental situation listed for the reference by the psychologist. The psychologist uses symbolic and typical data for machine learning. After the model is trained, the recognition accuracy will gradually improve. In the early period, the system will make a pre-judgment based on the existing research in psychology and issue a prompt to realize the function of finding out students with psychological problems on campus. The specific implementation content is described later. The following five functions belong to multiple threads.

\section{Screening students with psychological problems on campus}

\subsection{Based on the archive of online video surveil- lance and the function of facial detection and}




\section{snapshot}

All online surveillance cameras on the campus follow the Real Time Streaming Protocol, and the RFC2326 protocol pushes video images to the local network of the surveillance system. The network surveillance video recorder and the front-end server simultaneously pull streams from the local network to obtain real-time pictures; after the network surveillance video recorder obtains the video stream, the pictures of each station are separately stored in a five-minute cycle. The frontend primary and secondary processors are part of the front-end server, and the front-end primary processor performs real-time facial detection and snapshot. When a face is detected, it is captured at several frames per second, and the photos captured in the first-level processor are based on the SSIM algorithm in respective units $^{[10]}$. The screen of the highest rates is cropped to the facial area, and the shooting time and information of thee location are stored in files of image links. The front-end secondary processor calculates the relative position of space and the relative distance with others.

$$
\begin{gathered}
\sqrt[2]{\left(R x_{\mid}-L x_{\mid}\right)^{2}+\left(R y_{\mid}-L y_{\mid}\right)^{2}}=d \\
d_{1}-d_{2}<0
\end{gathered}
$$

1) Calculate the relative distance of the key points of the hips:

2) Compare the changes of the relative distance of hips in different images:

(If it is true, then it is in the direction of recess)

The SSIM algorithm is conducive to a function calculation. The formula is as follows: ( the average value of $\mathrm{X}$ is ${ }_{x}{ }_{x}$, the average value of $\mathrm{Y}$ is ${ }^{\mathrm{M}}{ }_{\mathrm{y}}$, the variation of $\mathrm{X}$ is $\sigma_{x}^{2}$, the variation of $\mathrm{Y}$ is $\sigma_{y}^{2}$, the co-variation of $\mathrm{X}$ and $\mathrm{Y}$ is $\sigma_{x y}$ )

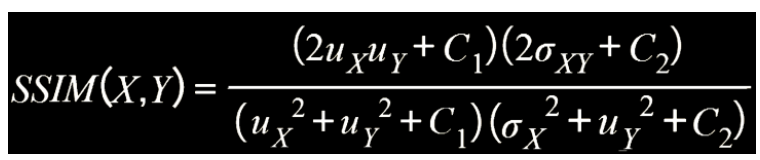

\section{2 $\mathrm{N}$ facial recognition and function of recogniz- ing facial emotions}

With the rapid development of technology, the model of facial recognition based on skin color or or multiHSMMs based on optical flow has become more mature ${ }^{[11-12]}$. In this paper, the method called the POST API is used. After acquiring the captured image of the face, the data is input to the "Detect API", and return to the face token data. This data is used to display the facial frame in real time; at the same time, the image is sent to the "Search API" port, and the port returns the face token data with the compared result of the character $=$, which refers to the user's name.

\subsection{Relative spatial position and moving direction based on recognition technology for human's key point}

Use the existing identification for human's key point SDK port to identify key points in the image. After inputting the image, the number of people and the coordinates of 14 main key points of each human are obtained, including the limbs, neck, nose, etc.

With a lot of experimental data, the hip is relatively stable when the person moves. The hip is one of the inactive bones that the human body cannot expand and displace greatly, and there will be no particularly large changes in appearance and size due to movement. Therefore, this paper defines the relative position and moving direction of the tested sample by comparing the changes of the hips in the picture.

\subsection{The function of restoring behaviour tracks with low sampling rate}

Select the time, place and moving direction of the person from the "Document of Image Feature" each time, where the person was photographed. Mark each photographed place on the map in chronological order, with the moving direction as data for reference.

After importing the map, a network is established based on walking restrictions for the overall route, and the position and perspective of the corresponding position in the map are calibrated. The overall network needs to include the possibility of each route, including but not limited to small routes and shortcuts.

Put the "Document of Image Feature" in units of individuals and place the location information of the camera. The relative moving direction is represented on the corresponding point in the form of 0 (progressive) and 1 (recessive). Connect two adjacent points in chronological order to restore the behaviour tracks. When the track is restored, the time sequence and the sequence of the behaviour direction are followed, such as on a straight line $\mathrm{d}$, with two points A and B. Points A and B are the starting and ending points of the sample. The sample was photographed by the monitor at point $\mathrm{A}$ at the first second and the monitor atpoint $\mathrm{B}$ at the eighth second. 


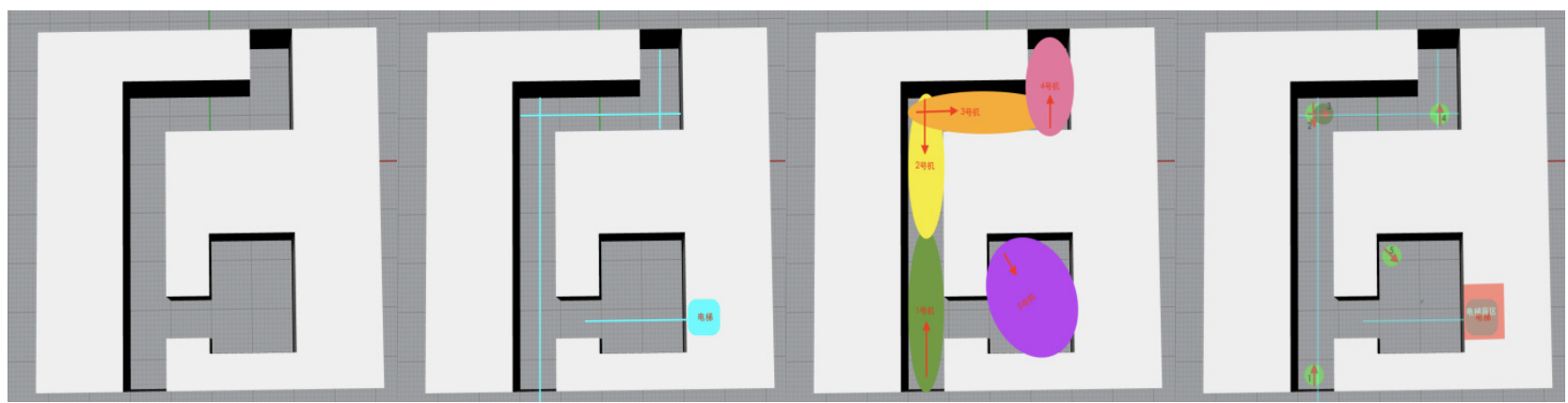

Figure 1. [Process 1 of restoring behaviour tracks]

When the moving track of the sample at A is recessive and the moving track at $B$ is progressive, the sample directly arrives from point $A$ to point $B$ through a straight line (represented by the green line). If the moving direction of the sample at $\mathrm{A}$ is recessive, and when $\mathrm{B}$ is progressive, the sample will detour around the line $\mathrm{d}$ to reach point $\mathrm{B}$ (red line). The restored behaviour track will be saved with the targeted descriptive file.

\subsection{Deduce samples at high risk of suicide based on weighted calculations in psychology}

The weighted calculation based on psychology which deduces the samples at high risk of suicide refers to the model of recognizing suicide ideas. Existing research results in psychology have shown that depressive factors have a significant effect on predicting suicide, and the suicide risk increases with the level of depression ${ }^{[13]}$. With the accumulation of psychological problems, the samples will reflect in many aspects, such as expression and behavior.

This paper has completed the data collection of the sample's facial recognition, emotional analysis, and behavior tracking, and recognizing the suicide intention.

According to the above information, a rule of weighted calculation is defined. When the cumulative amount of samples reaches a certain level, the system will issue a warning, which is fed back by the supporting machine (SVM).

Set the variable of mental symptom, initially set the variable of the negative emotional expression each time. The positive variable of emotional expression decrease by one, based on the restoration system of the behaviour tracks when the deviation from the normal behaviour is detected. The variable increases by one every 6 hours. The number of positive emotional expressions recognized by the hour system is less than onethird of the number of that of plain facial expressions (no expressions), recognized by the variable plus one ${ }^{[14]}$. The system is in a preliminary state of being designed, and the specific weighted ratio is adjusted according to the actual operation.

\subsection{Machine learning based on supporting vector machines.}

The screening of high-risk samples on campus suicide is mainly by restoring the sample's behavior tracks within a specific time, that is, the evaluation of facial expressions at several consecutive times and spatial positions into a continuous track in time. In this paper, we will analyze the targeted sentiment of each point and establish a super-dimensional point according to the number of variables, and identify its behavioral tracks through the SVM to achieve screening.

This paper is based on a large number of existing SVM models, and adjusts a set of efficient supporting vector machine $\mathrm{SVM}^{[15-21]}$. Supporting vector machines can effectively carry out recognition tasks in the case of non-linearity, small sample, and many modeling dimensions, and can be generalized to other machine learning problems such as function regression. For super-dimensional points arbitrarily distributed in a super-dimensional space (N-dimensional), find a super-dimensional surface (N-1 dimension), also known as a decision boundary, so that the super-dimensional point can be completely divided into a single type of point.

It is necessary to map the data of the SVM classifier to the targeted high-dimensional space with a kernel function to make it easy to distinguish. Common kernel functions include linear kernel functions, polynomial kernel functions, Gaussian kernel functions, etc.

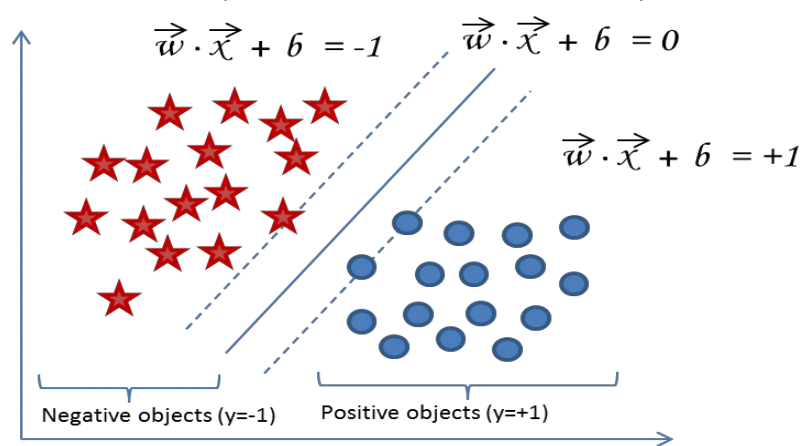


For the analysis of parameter in SVM., each data point is a facial expression characteristic of a sample at specific time. This paper mainly gives each data point three characteristic behaviour tracks, facial emotions, and status of the group.

In this paper, Gaussian (RBF) is set as a kernel function of machine learning. The Gaussian radial basis function is a highly localized kernel function, which can reflect a sample to a higher dimensional space, suitable for a wider range of samples, and is suitable for the case of fewer variables.

$$
\kappa\left(x, x_{i}\right)=\exp \left(-\frac{\left\|x-x_{i}\right\|^{2}}{\delta^{2}}\right)
$$

Gamma is the main variable in the Gaussian kernel function, which affects the spatial distribution after reflecting data. The variable of gamma provides a specific location for the SVM. The value of the factor in the experiment determines the degree of the factor. Small factor of little value will slower the learning speed and higher the rate of errors, otherwise, it will affect the normal fitting of the classifier system.

\section{Conclusion}

This paper proposes recognizing facial expressions by machine learning, and collecting data through the monitoring system on campus, with the computer processing of a large amount of information and fast speed. The traditional self-rating scale takes a long time to collect and is random, so the result is inaccurate. It can only predict the suicidal psychology of the respondents. The monitoring system for psychological problems proposed in this paper has an unbelievable marketing prospect. Further comprehensive analysis through multi-dimension samples can bring more satisfying results, thereby greatly preventing and reducing campus suicide and crime rates.

After experimental verification, the model proposed in this paper is feasible. (specifically shown in the above results) However, this study still exists deficiencies, such as the lack of dimension, from only visual monitoring. For further application, monitoring for online behaviour such as issuing public opinions on social platforms can be added; there is no standard for the determination of suicide intentions. We need many suicide cases for machine learning but not only the existing studied results to effectively and correctly predict suicide ideas.

How do I plan to optimize the study after this? Eventually, the system can be used not only for predicting campus suicide behaviour, but also for violent behaviour and dynamic personnel management.

\section{References}

[1] Sun Y, Tao FB, Gao M. A study on the relationship between suicide behavior and psychological factors of middle school students in Hefei[J]. Chinese Journal of Epidemiology, 2006: 27(1): 33-36.

[2] Xing Y, Ji CY, Ji H. The prevalence of injuring behaviors among middle school students in Shijingshan District, Beijing[J]. Chinese School Health, 2003, 24(1): 28-29.

[3] Liang JL, Sun Lu, Zhao JB. Analysis on the incidence and influential factors of suicide ideas among middle school students [J]. Journal of Clinical Psychiatry, 2000, 10(3): 144-146.

[4] Zhang ZQ, Guo LT. A survey on the current status quo of suicide attempts among middle school students in urban areas of Chengdu [J]. Chinese Journal of Epidemiology, 2003, 24(3): 189-191.

[5] Luo CY, Peng NN, Zhu W. Study on the status quo of adolescents' high-risk behaviors of in Shanghai (5) [J]. Chinese Journal of School Doctor, 2003, 17(3): 197-199.

[6] Goldstona DB,Beth A. Reboussinb and Stephanie Sargent Pre-dictors of Suicide A tempts State and Trait Components[J]. Journalof A abnormal Psychology, 2006, 115(4): 842-849.

[7] Wu LH. A model for predicting college students' suicide tendency by mental health symptoms[J]. Chinese Journal of Health Psychology, 2010(18): 99-100.

[8] Li XY. Extraction and recognition of expressions based on sequence images[J]. Dalian University of Technology, 2006.

[9] Gao HY. Adolescent suicide[J]. Chinese Journal of Practical Pediatrics, 2007, 22(3): 169-172.

[10] Ying Y, Duan MA. Jian-Hua, and C. Wu-Fan. "Improved SSIM medical image quality assessment." Computer Engineering and Applications (2010).

[11] Miao Shaojun. Facial expression recognition based on optical flow and multi-HSMMs that face can be hidden. Jiangsu University, 2008.

[12] Wang L. Research on automatic extraction and tracking technology of facial expressions. Diss. Hunan University, 2007.

[13] Jin SH. "Study on predicting adolescents' suicide intention." Youth Research, 1996(5): 40-45.

[14] Ding N. "Prediction and analysis of college students' suicide based on machine learning." Modern Electronic Technology, 2017(21): 99-101 + 105 .

[15] Mathur A, Foody GM. Multiclass and binary SVM classification: Implications for training and classification users[J]. IEEE Geoscience and remote sensing letters, 2008, 5(2): 241245.

[16] Hu W, Tan T, Wang L, Maybank S. A survey on visual surveillance of object motion and behaviors. IEEE Transactions on Systems, Man, and Cybernetics, Part C (Applications and Reviews), 2004, 34(3): 334-352.

[17] Shan CF, Gong SG, Peter WM. Facial expression recognition based on local binary patterns: A comprehensive study[J]. Image and vision Computing, 2009, 27(6): 803-816.

[18] Keerthi SS, Chih-Jen L. Asymptotic behaviors of support vector machines with Gaussian kernel[J]. Neural computation 15, 2003(7): 1667-1689.

[19] Wöllmer M, Angeliki M, Florian E, et al. Context-sensitive multimodal emotion recognition from speech and facial expression using bidirectional 1stm modeling[J]. In Proc. Interspeech 
2010, Makuhari, Japan, pp. 2362-2365. 2010.

[20] Ryoo MS. Human activity prediction: Early recognition of ongoing activities from streaming videos[C]. In Computer Vision (ICCV), 2011,IEEE International Conference on, pp. 1036-
1043. IEEE, 2011.

[21] Wang XG. Intelligent multi-camera video surveillance: A review. Pattern recognition letters 34, 2013(1): 3-19. 